\title{
Effects of nitrogen supply level on photosynthesis and chlorophyll fluorescence characteristics of rice under salt stress
}

\author{
Chen $\mathrm{Xu}^{1 \dagger}$, Qian $\mathrm{Li}^{2 \dagger}$, Xiaolong $\mathrm{Liu}^{3}$, Hongjun Wang ${ }^{2}$, Xuanhe Liang ${ }^{2}$, Fenglou Ling ${ }^{1}$, Zhihai $\mathrm{Wu}^{1}$, \\ Zhian Zhang ${ }^{1 *}$, Zhanyu Chen ${ }^{1 *}$ \\ ${ }^{1}$ Department of Agronomy, Jilin Agricultural University, Changchun, P.R. China, ${ }^{2}$ Department of Agricultural Resources and Environment, \\ Jilin Academy of Agricultural Sciences, Changchun, P.R. China, ${ }^{3}$ College of Life Sciences, Resources and Environment sciences, Yichun \\ University, Yichun, P.R. China \\ tThese authors have contributed equally to this work.
}

\section{A B S TR A C T}

The change of photosynthesis and chlorophyll fluorescence parameters of rice were studied in five nitrogen levels during tillering, booting, and heading periods under salt stress. The net photosynthetic rate $\left(P_{\mathrm{N}}\right)$, stomatal conductance $\left(\mathrm{g}_{\mathrm{s}}\right)$, transpiration rate $(E)$, apparent mesophyll conductance $(A M C)$, effective quantum yield of PSII photochemistry $\left(\Phi_{P S I I}\right)$, electron transport rate (ETR), and photochemical quenching coefficient $\left(\mathrm{q}_{\mathrm{p}}\right)$ were significantly declined under salt stress and exhibited a lower magnitude of decline in the $2 \mathrm{~N}, 1 \mathrm{~N}$, and $1 / 2 \mathrm{~N}$ treatments respectively during tillering, booting, and heading periods. The stomatal limit value $\left(L_{s}\right)$, Nonphotochemical quenching (NPQ) and plasma membrane permeability were significantly increased and exhibited a lower magnitude of increase in the $2 \mathrm{~N}, 1 \mathrm{~N}$, and $1 / 2 \mathrm{~N}$ treatments respectively during tillering, booting, and heading periods. The amount of nitrogen in the nutrient solution should be reduced $50 \%$ after the heading period to decrease salt damage to rice under salt stress.

Keywords: Chlorophyll fluorescence; Nitrogen level; Photosynthetic characteristics; Rice; Salt stress

\section{INTRODUCTION}

As one of the most important food crops, rice (Oryza sativa L.) is widely grown in China. China ranked second in the world in rice planting area, first in total output and third in average yield per unit area in the year of 2018. Rice is mostly grown in saline soil in arid and semiarid areas in the western part of Jilin Province, in which crop growth is difficult. Rice, more than other crops, has been planted in a much larger area in recent years. Meanwhile, the probability of salt damage to rice has increased greatly. There is hope that rice cultivation can be improved in the cropland in this area and rice yield can be increased. The main areas that have been subjected to expanding rice planting are in the western region of Jilin Province as part of the implementation of the project "Bring the Nen River into the Baicheng Area" for the past several years. However, due to the large areas of saline-alkali land in this region, the normal production of rice is seriously restricted. Soil salt concentrations can be as high as $0.5 \%$ to $1 \%$ in this region, making it difficult to grow crops (Chi and Wang, 2010). The salt concentration in the soil of rice growing areas can reach $0.1 \%$ to $0.3 \%$. The influence of salt stress on crops was mainly reflected in ion poisoning (Summart et al., 2010) and osmotic stress (Serrano et al., 1999; Ashraf and Sultana, 2000; Munns and Tester, 2008). The accumulation of $\mathrm{Na}^{+}$and $\mathrm{Cl}^{-}$in the plant leaves eventually leads to irreversible changes in the plasma membrane permeability of the leaf cell. Too great a salt level could cause improper absorption of water and nutrients (Golldack et al., 2014). Photosynthesis is indispensable to plant growth. Plants might get the nutrients they need from photosynthesis. Salt stress could damage the photosynthetic system of plants by forcing a decrease of the photosynthetic capacity of plant leaves (Cha-Um et al., 2010; Chaum and Kirdmanee, 2009) and damage to the photosynthetic system II (Lu et al., 2002; Netondo et al., 2004).

\footnotetext{
${ }^{*}$ Corresponding author:

Zhian Zhang and Zhanyu Chen, Department of Agronomy, Jilin Agricultural University, Changchun, P.R. China.

E-mail: zhangzhian6412@163.com/chenzhanyu2000@sina.com.
}

Received: 21 May 2019; $\quad$ Accepted: 30 September 2019 
Nitrogen has an important regulatory effect on crop resistance (Gamett et al., 2009). The nitrogen content in the crop changes under the action of salt. Salt has a negative correlation effect on the formation of ammonium nitrogen in rice in the seedling stage. The excessive accumulation of ammonium nitrogen is due to the increase of sodium ions in rice, thus exacerbating the damage caused by salt stress (Hoai et al., 2003). Chakrabarti and Mukherji, (2003) found that salt stress could reduce the nitrogen content of mungbean plants and interfere with the nitrogen metabolism process of mungbean. Nitrogen is one of the nutrients that plays a key role in increasing crop yield during crop growth and has an important regulatory effect on crop resistance (Silveira et al., 2001). The suitable nitrogen level is beneficial to the regulation of plants against salt stress. Research on chickpea (Cicer arietinum) showed that low nitrogen levels could alleviate the influence of salt stress on the photosynthetic rate of the leaves, while high nitrogen levels would aggravate the adverse effect of salt stress on the photosynthesis of the leaves. The photosynthetic rate of chickpea leaves cultured at a normal nitrogen level was higher than that at a high nitrogen level (Soussi et al., 1998). The study of Liu et al. (2015) showed that during rice booting period, the factors influencing the photosynthesis of rice leaves under salt stress at low nitrogen level mainly came from non-stomatal restrictions, while at high nitrogen level, stomatal restrictions were added except stomatal restrictions.

At present, there have been few reports on the effect of nitrogen level on photosynthesis and chlorophyll fluorescence characteristics of rice at different development periods under salt stress. Two japonica rice varieties which were widely cultivated in Jilin Province were selected for use in this study. A study on the influence of photosynthetic and chlorophyll fluorescence characteristics of rice under salt stress during the tillering, booting, and heading periods for five nitrogen levels was conducted. The optimum nitrogen supply level of rice in each period is discussed. This work provides a theoretical basis for the study of rice production and salt tolerance in Jilin Province, China.

\section{MATERIALS AND METHODS}

\section{Characteristics of rice used in the study}

Changbai 9 and Jinongda 19, two varieties of conventional japonica rice (Oryza sativa subsp. japonica) grown in northern China, were used as experimental materials. Both varieties are medium early maturity varieties, and their growth period is approximately $135 \mathrm{~d}$. Changbai 9 is a salt-tolerant variety, and Jinongda 19 is a salt-sensitive variety. The two varieties were selected from 112 rice varieties grown in Jilin Province by means of IRRI Yoshida (1976) nutrient solution improved with $\mathrm{NaCl}$ with a concentration of $80 \mathrm{mmol} / \mathrm{L}$ during seed germination and the seedling period. Our group has conducted previous studies on the physiological response mechanism of rice under different salt concentration (Liu et al., 2015). We found that when the concentration of nutrient solution salt in rice reached $80 \mathrm{mmol} / \mathrm{L}$ under the control condition, it was the critical state of the response of rice to salt stress. When the salt concentration in the nutrient solution was lower than $80 \mathrm{mmol} / \mathrm{L}$, the effect of salt stress on the physiological mechanism of rice was not significant, and the change rule of each index could not be found. When the salt concentration in nutrient solution was higher than $80 \mathrm{mmol} / \mathrm{L}$, the salt stress would dramatically damage the normal growth and development of rice, and the response mechanism of different nitrogen levels to the physiological characteristics of rice under salt stress could not be determined.

\section{Experimental design and treatments}

The test was carried out in plant growth chambers with 10 hours of light each day. The day and night temperatures were $23 \pm 2^{\circ} \mathrm{C}$ and $17 \pm 2^{\circ} \mathrm{C}$, respectively, and the humidity was $60 \pm 5 \%$. Rice germination was carried out in a culture dish. The plants were transplanted into buckets (diameter, $35 \mathrm{~cm}$; height, $45 \mathrm{~cm}$ ) when the plant height was greater than $10 \mathrm{~cm}$. The roots of the seedlings were fixed with absorbent cotton and inserted into a perforated black plastic foam plate. The plant spacing was $5 \mathrm{~cm}$ with eight plants per bucket. One-hundred eight buckets were used for the test. Among them, ninety buckets of improved IRRI Yoshida (1976) nutrient solution were cultured with 1/4, 1/2, 1, 2 and 4 times of normal nitrogen content. Eighteen buckets were selected to each nitrogen level. The remaining eighteen buckets were selected to control group and the improved IRRI Yoshida (1976) nutrient solution with normal nitrogen level was applied. The nitrogen in nutrient solution was in the form of ammonium nitrate nutrient solution. The normal nitrogen level in the nutrient solution was 0.715 $\mathrm{mmol} / \mathrm{L}$. The nitrogen levels, which were $4,2,1 / 2$, and $1 / 4$ times the normal nitrogen level in the nutrient solution were $2.86,1.43,0.3575$, and $0.17875 \mathrm{mmol} / \mathrm{L}$, respectively. All of the nutrient solutions had a $\mathrm{pH}$ value of $5.1 \pm 0.2$. The nutrient solution was replaced once every seven days. At the same time, the $\mathrm{pH}$ value of the nutrient solution needed to be adjusted to remain within $5.1 \pm 0.2$. The treatment was carried out for seven days at each growth period. Samples were taken on the $8^{\text {th }}$ day after the treatment began. The image of the treatment is shown in Fig. 1, which shows the growth and development of rice under salt stress.

\section{Method for determination of photosynthetic parameters} The determination of photosynthetic characteristics was carried out using a Li-6400 portable photosynthetic 


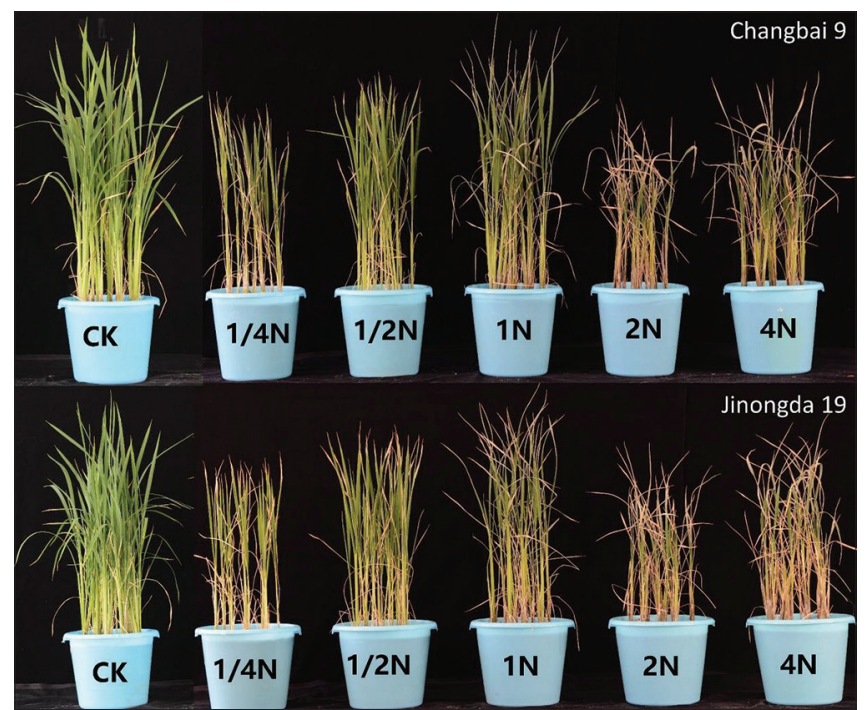

Fig 1. Growth and development of rice under salt stress.

measurement system (Li-Cor Biosciences, Lincoln, NE, USA). The photosynthetic parameters were determined by the method of (Brandner and Salvucci, 2002). Three flag leaves were randomly selected in each bucket for determination at the tillering period, and ear leaves were selected at the booting and heading periods.

\section{Method for determination of chlorophyll fluorescence parameters}

The determination of chlorophyll fluorescence was carried out using the Li-6400 portable photosynthetic measurement system. The chlorophyll fluorescence parameters were determined by the method of (Brandner and Salvucci, 2002). The measured position of the leaf was the same as that of photosynthesis.

\section{Data analysis}

All data were analyzed statistically using Microsoft Excel 2013 and SAS 9.0 software. Significance probability was used to test the significance of each treatment.

\section{RESULTS}

\section{Photosynthetic traits}

As can be seen in Fig. 2, the $P_{\mathrm{N}}$ of the leaves of the two rice varieties under salt stress was significantly lower than that of the control. The salt-tolerant rice variety Changbai 9 exhibited a lower magnitude of decline than that of salt-sensitive rice variety Jinongda 19. Compared with the control, the $P_{\mathrm{N}}$ for the $2 \mathrm{~N}$ treatment exhibited a lower magnitude of decline during the tillering period under salt stress. There was no significant difference between the $1 \mathrm{~N}$ treatment and $2 \mathrm{~N}$ treatment of Changbai 9 . The $P_{\mathrm{n}}$ of Changbai 9 of $2 \mathrm{~N}$ treatment decreased by $38.84 \%$, and that of Jinongda 19 decreased by $55.53 \%$. The $P_{n}$ of Changbai 9
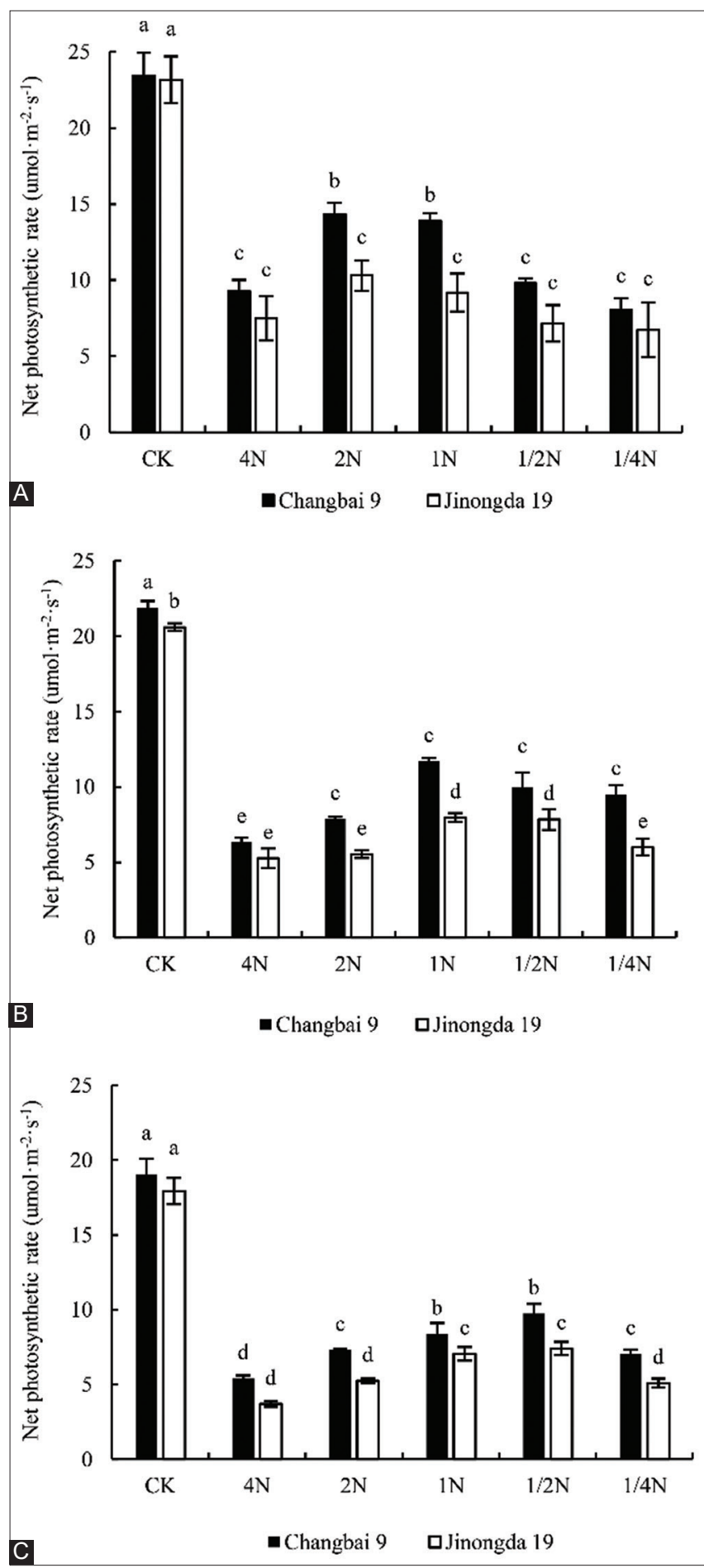

Fig 2. Effect of salt stress on net photosynthetic rate of rice leaves for different nitrogen levels at different growth periods (A) Tillering period; (B) Booting period; (C) Heading period. Different lowercase letters indicate significant differences $(P<0.05)$.

of $1 \mathrm{~N}$ treatment decreased by $40.67 \%$. The $P_{\mathrm{N}}$ exhibited a lower magnitude of decline for the $1 \mathrm{~N}$ treatment during the booting period under salt stress. There was no significant difference between the $1 \mathrm{~N}$ treatment and $1 / 2 \mathrm{~N}$ treatment of Jinongda 19. The $P_{\mathrm{N}}$ of Changbai 9 and Jinongda 19 decreased by $46.57 \%$ and $61.31 \%$, respectively. The $P_{\mathrm{n}}$ 
for the $1 / 2 \mathrm{~N}$ treatment exhibited a lower magnitude of decline during the heading period under salt stress. The $P_{n}$ of Changbai 9 decreased by $48.89 \%$ and that of Jinongda 19 decreased by $58.73 \%$.

Fig. 3 shows the effect of nitrogen supply level on $g_{\mathrm{s}}$ and AMC of rice leaves under salt stress during different growth periods. The $g_{\mathrm{s}}$ and AMC of both rice varieties both showed a downward trend under salt stress, and the differences were significant. Compared with the control, the parameter changes in the three periods were different. The $g_{\mathrm{s}}$ and AMC of the $2 \mathrm{~N}$ treatment exhibited a lower magnitude of decline on the tillering period. Changbai 9 decreased by $28.41 \%$ and $35.85 \%$, and Jinongda 19 decreased by $30.69 \%$ and $53.62 \%$, respectively. In the booting period, the $g_{\mathrm{s}}$ and AMC exhibited a lower magnitude of decline in the $1 \mathrm{~N}$ treatment; Changbai 9 decreased by $25.71 \%$ and $42.35 \%$, respectively, while Jinongda 19 decreased by $32.19 \%$ and $57.95 \%$, respectively. In the heading period, the $g_{\mathrm{s}}$ and AMC exhibited a lower magnitude of decline in the $1 / 2 \mathrm{~N}$ treatment; Changbai 9 decreased by $65.97 \%$ and $45.19 \%$, respectively, while Jinongda 19 decreased by $67.97 \%$ and $56.38 \%$, respectively.

Fig. 4 shows that the $E$ of the two rice varieties showed a downward trend and all the differences reached a significant level under salt stress. Compared with the control, the Exhibited a lower magnitude of decline in the $2 \mathrm{~N}$ treatment during the tillering period. Changbai 9 and Jinongda 19 decreased by $21.65 \%$ and $38.82 \%$, respectively. In the booting stage, the $E$ exhibited a lower magnitude of decline in the $1 \mathrm{~N}$ treatment, and the two
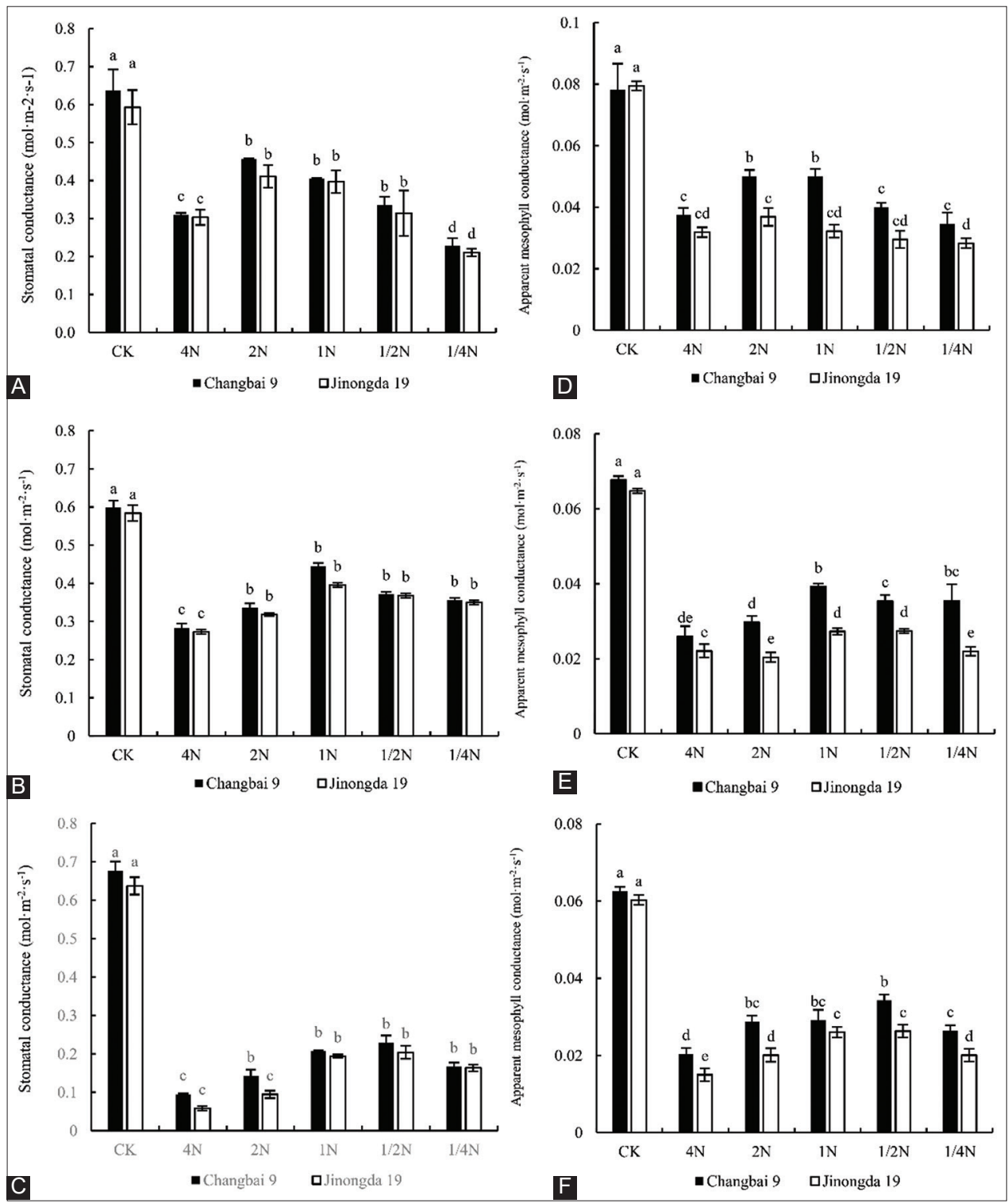

Fig 3. Effect of salt stress on stomatal conductance and apparent mesophyll conductance of rice leaf for nitrogen levels at different periods (A,C) Tillering period; $(B, E)$ Booting period; $(C, F)$ Heading period. Different lowercase letters indicate significant differences $(P<0.05)$. 

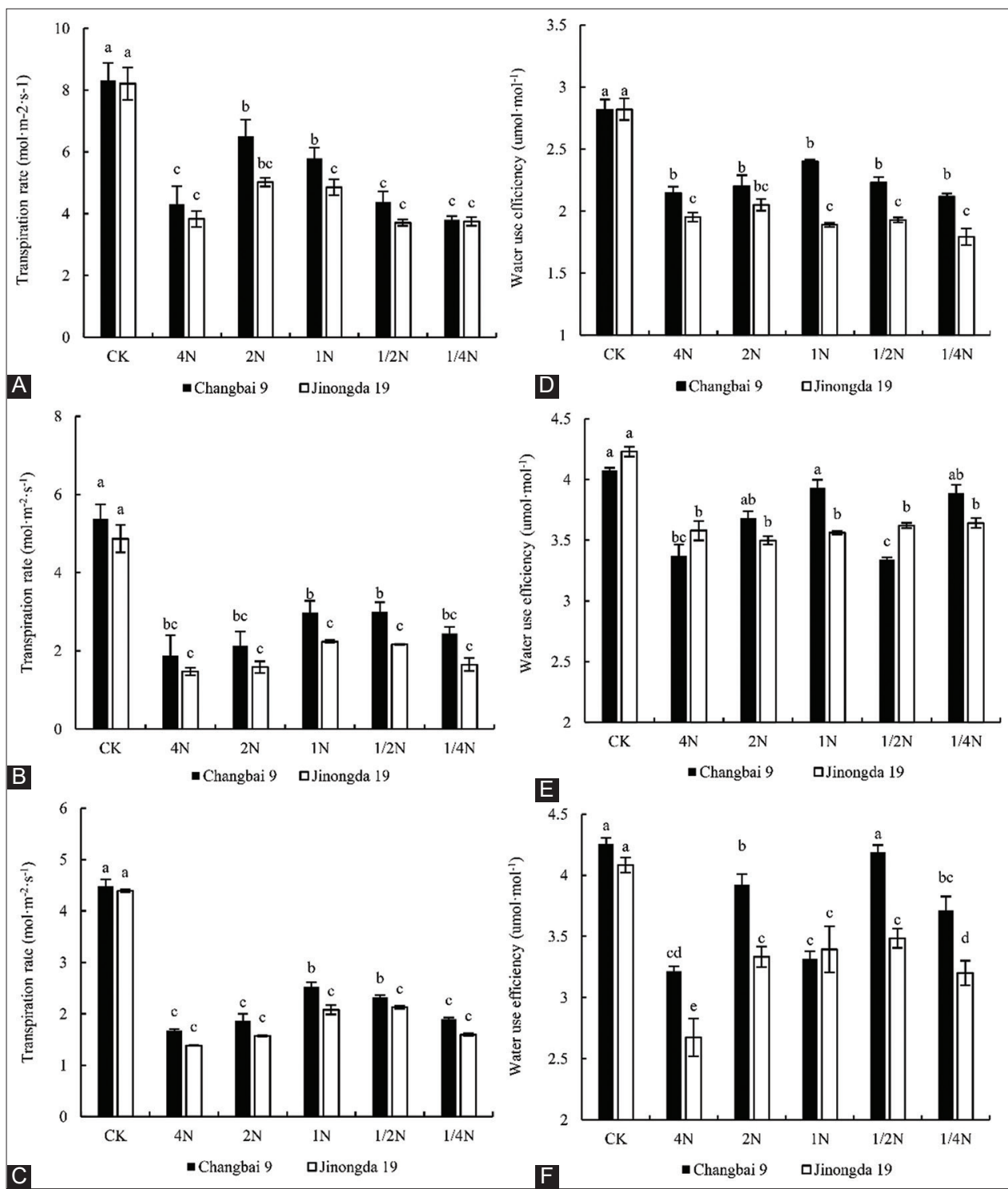

Fig 4. Effect of salt stress on transpiration rate and water use efficiency of rice leaves for nitrogen levels during different growth periods (A,C) Tillering period; $(B, E)$ Booting period; $(C, F)$ Heading period. Different lowercase letters indicate significant differences $(P<0.05)$.

varieties decreased by $44.64 \%$ (Changbai 9) and 54.02\% (Jinongda 19), respectively. In the heading period, the Changbai 9 of E exhibited a lower magnitude of decline in the $1 \mathrm{~N}$ treatment with a decrease of $43.52 \%$. The $E$ of Jinongda 19 exhibited a lower magnitude of decline in the $1 / 2 \mathrm{~N}$ treatment with a decrease of $51.64 \%$.

The WUE of rice leaves of the two rice varieties showed a downward trend under salt stress. Changbai 9 decreased less than Jinongda 19. Compared with the control, the WUE of the leaves of Changbai 9 in the $1 \mathrm{~N}$ and $2 \mathrm{~N}$ treatments had no significant difference during the booting and heading periods. Therefore, it is speculated that appropriate nitrogen levels can reduce the decrease of WUE at different growth periods, and higher WUE could guarantee better progress of photosynthesis in rice leaves.
Fig. 5 shows that the $L_{\mathrm{s}}$ and $C_{\mathrm{i}}$ of rice leaves had an upward trend in the $2 \mathrm{~N}$ treatment during the tillering period, and increased by $15.71 \%$ and $22.83 \%$ for Changbai 9 and Jinon and downward trend, respectively, under salt stress. Compared with the control, the $L_{\mathrm{s}}$ exhibited a lower magnitude of increase in Changbai 9 and it was the smaller than Jinongda 19. In the booting period, the $L_{\mathrm{s}}$ exhibited a lower magnitude of increase in the $1 \mathrm{~N}$ treatment, increasing by $31.03 \%$ (Changbai 9) and 32.88\% (Jinongda 19). At the heading period, the $L_{\mathrm{s}}$ exhibited a lower magnitude of increase in the $1 \mathrm{~N}$ treatment, increasing by $19.91 \%$ (Changbai 9) and $22.70 \%$ (Jinongda 19). Compared with the control, the $C_{\mathrm{i}}$ in rice leaves exhibited a lower magnitude of deline during the tillering, booting, and heading periods in the $2 \mathrm{~N}, 1 \mathrm{~N}$, and $1 / 2 \mathrm{~N}$ treatments, respectively. Therefore, it is speculated that with the increase of nitrogen 


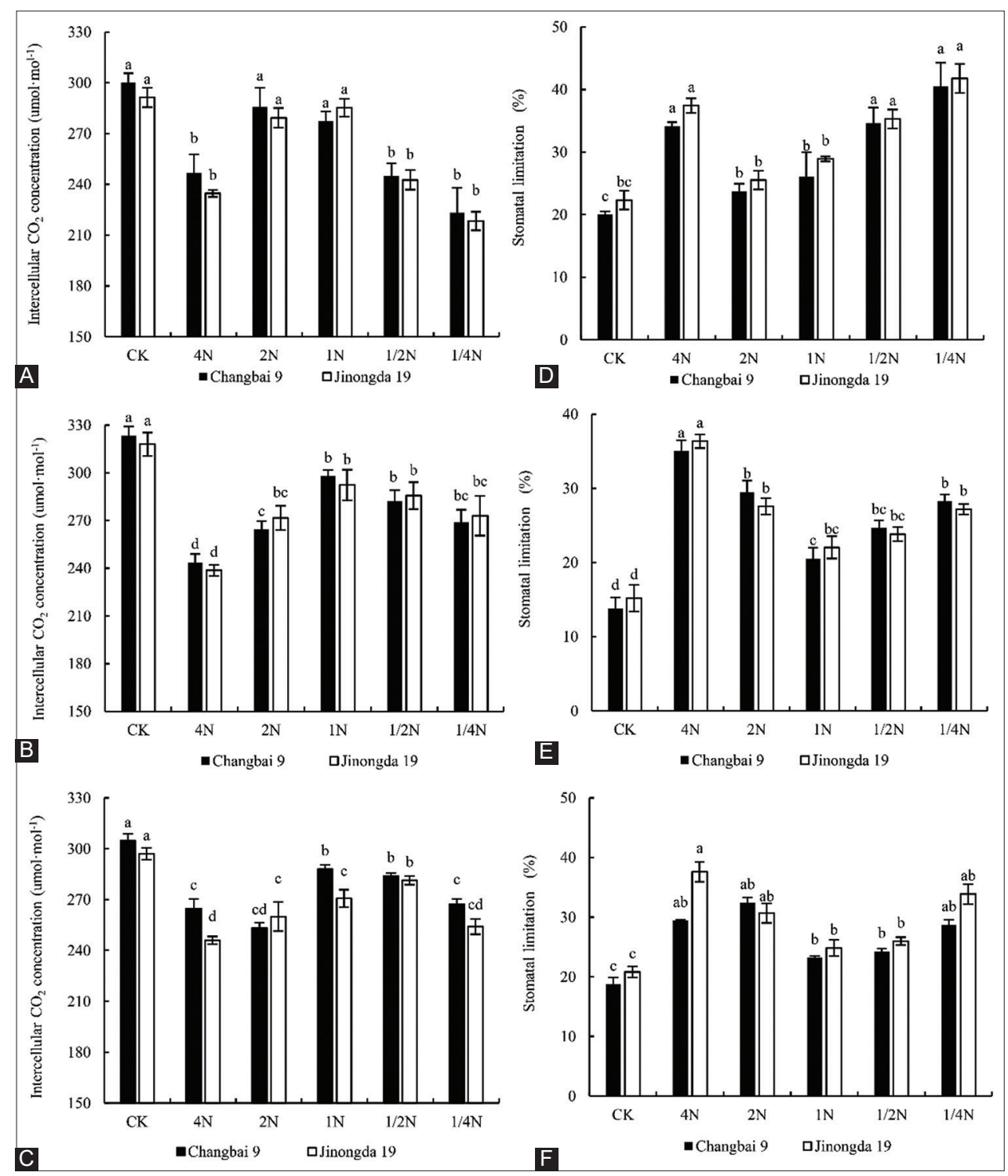

Fig 5. Effect of salt stress on intercellular $\mathrm{CO}_{2}$ concentration and stomatal limitation of rice leaves for nitrogen levels at different growth periods $(A, C)$ Tillering period; $(B, E)$ Booting period; $(C, F)$ Heading period. Different lowercase letters indicate significant differences $(P<0.05)$.

concentration, stomatal restriction was increasing, and the reason for the decrease of the photosynthetic rate might come from stomatal restriction. With the growth and development of rice, the decrease of the nitrogen level was beneficial to the photosynthesis of rice leaves under salt stress, enhancing the ability of rice plants to resist salt damage.

\section{Chlorophyll fluorescence characteristics}

As shown in Fig. 6, the $\mathrm{F}_{\mathrm{v}} / \mathrm{F}_{\mathrm{m}}$ of the leaves of two rice varieties declined. Compared with the control, the $\mathrm{F}_{\mathrm{v}} / \mathrm{F}_{\mathrm{m}}$ of rice leaves exhibited a lower magnitude of decline in the $2 \mathrm{~N}$ treatment during the tillering period. Changbai 9 and Jinongda 19 decreased by $4.73 \%$ and $4.10 \%$, respectively. In the booting period, the $\mathrm{F}_{\mathrm{v}} / \mathrm{F}_{\mathrm{m}}$ exhibited a lower magnitude of decline in the $1 \mathrm{~N}$ treatment, and
$\mathrm{F}_{\mathrm{v}} / \mathrm{F}_{\mathrm{m}}$ decreased by $2.14 \%$ (Changbai 9) and 10.59\% (Jinongda 19), respectively. In the heading period, a clear change in $\mathrm{F}_{\mathrm{v}} / \mathrm{F}_{\mathrm{m}}$ of Changbai 9 was not seen with the increase in nitrogen concentration. Compared with the control, the difference was significant only at the $4 \mathrm{~N}$ treatment.

Fig. 7 shows that the $\Phi_{\text {PSII }}$ and ETR of both rice varieties declined under salt stress. In addition to Changbai 9, which had no significant difference compared with the control, all of the other treatments reached a significant level during the tillering period. The $\Phi_{\text {PSII }}$ and ETR of both rice varieties leaves exhibited a lower magnitude of decline in the $2 \mathrm{~N}$ treatment under salt stress during the tillering period. Changbai 9 decreased by $5.72 \%$ and $2.01 \%$, respectively, and Jinongda 19 decreased by 

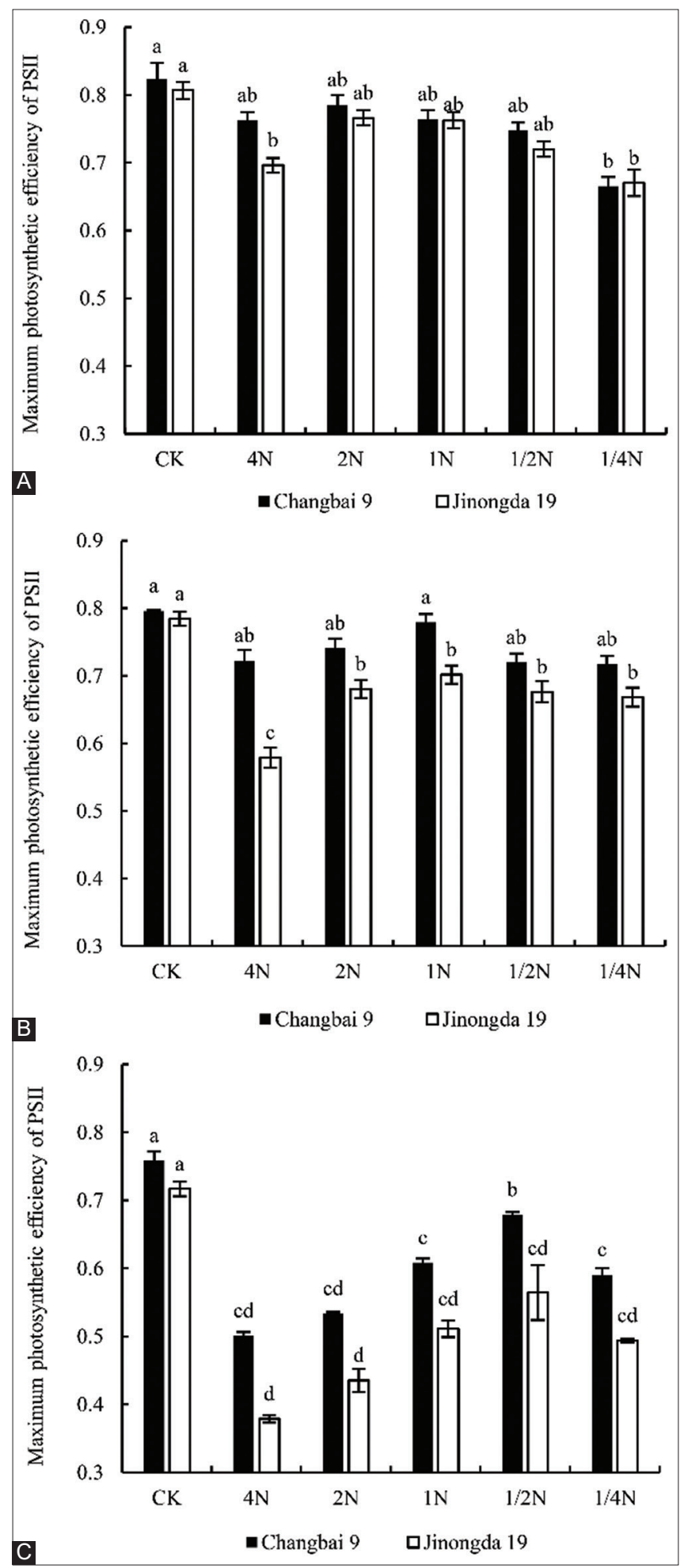

Fig 6. Effect of salt stress on maximum photosynthetic efficiency of PSII of rice leaves for different nitrogen levels during different growth periods (A) Tillering period; (B) Booting period; (C) Heading period. Different lowercase letters indicate significance differences $(P<0.05)$.

$15.43 \%$ and $3.23 \%$, respectively. In the booting period, two parameters exhibited a lower magnitude of decline in the $1 \mathrm{~N}$ treatment. Changbai 9 decreased by $24.73 \%$ and $16.31 \%$, respectively, and Jinongda 19 decreased by
$40.45 \%$ and $22.84 \%$, respectively. In the heading period, two parameters exhibited a lower magnitude of decline in the $1 / 2 \mathrm{~N}$ treatment. Changbai 9 decreased by $23.36 \%$ and $20.43 \%$, respectively, and Jinongda 19 decreased by $20.97 \%$ and $27.56 \%$, respectively. The results show that the photosynthetic system II of rice leaves was impaired under salt stress. That, in turn, led to $\Phi_{\mathrm{PSII}}$ and ETR being reduced, while the appropriate nitrogen concentration may help the rice leaves to protect the photosynthetic system II.

As shown in Fig. 8, $\mathrm{q}_{\mathrm{p}}$ of the two rice varieties showed a downward trend under salt stress, while NPQ showed an upward trend under salt stress. Compared with the control, all of the increases in NPQ reached a significant level. The NPQ of Changbai 9 and Jinongda 19 in the $2 \mathrm{~N}$ treatment exhibited a lower magnitude of increase during the tillering period, increasing by $4.75 \%$ and $6.11 \%$ respectively. In the booting period, the NPQ for the two varieties exhibited a lower magnitude of increase in the $1 \mathrm{~N}$ treatment, increasing by $11.55 \%$ (Changbai 9) and $12.46 \%$ (Jinongda 19). In the heading period, the NPQ of the two varieties exhibited a lower magnitude of increase in the $1 / 2 \mathrm{~N}$ treatment were the smallest, with $6.79 \%$ for Changbai 9 and $8.54 \%$ for Jinongda 19. The NPQ of the two varieties exhibited a higher magnitude of increase in the $4 \mathrm{~N}$ level during the heading period. Changbai 9 increased by $16.09 \%$, and Jinongda 19 increased by $17.67 \%$. It is speculated that salt stress may cause rice leaves to consume more energy through heat dissipation to resist salt damage.

\section{DISCUSSION}

\section{Photosynthesis}

Many studies have shown that salt stress leads to the decrease in the $P_{\mathrm{N}}$ of the crop leaves and ultimately, the decrease in rice yield (Chaves et al. 2009), and it was mainly attributed to the joint effect of stomatal and non-stomatal restrictions (Parida and Das 2005; da Silva et al. 2008). A study by Siddiqui et al (2010) on brassica (Blassikakapestris) showed that the low nitrogen can alleviate the effect of salt stress on leaf photosynthesis and reduce the decrease of $P_{\mathrm{N}}$ and $g_{\mathrm{s}}$. The main reason for the decrease in the photosynthetic rate at the low nitrogen level was determined to be non-stomatal restriction under salt stress. Research by Ali-Dinar et al. (1999) on guava (Psidium guajava) showed that a low concentration of nitrogen can help alleviate the decrease of $P_{n}$ in guava as well as improve the closure of the stomata of guava leaves. The results of this study indicated that salt stress resulted in a significant decrease in the $P_{\mathrm{N}}, g_{\mathrm{s}}, C_{\mathrm{i}}$, and $E$ of rice leaves $(\mathrm{P}<0.05)$. The percent decreases of $P_{\mathrm{N}}, g_{\mathrm{s}}, C_{\mathrm{i}}$, and $E$ of rice leaves in the $2 \mathrm{~N}, 1 \mathrm{~N}$, and $1 / 2 \mathrm{~N}$ treatments were the smallest during the tillering, booting, and heading periods, 

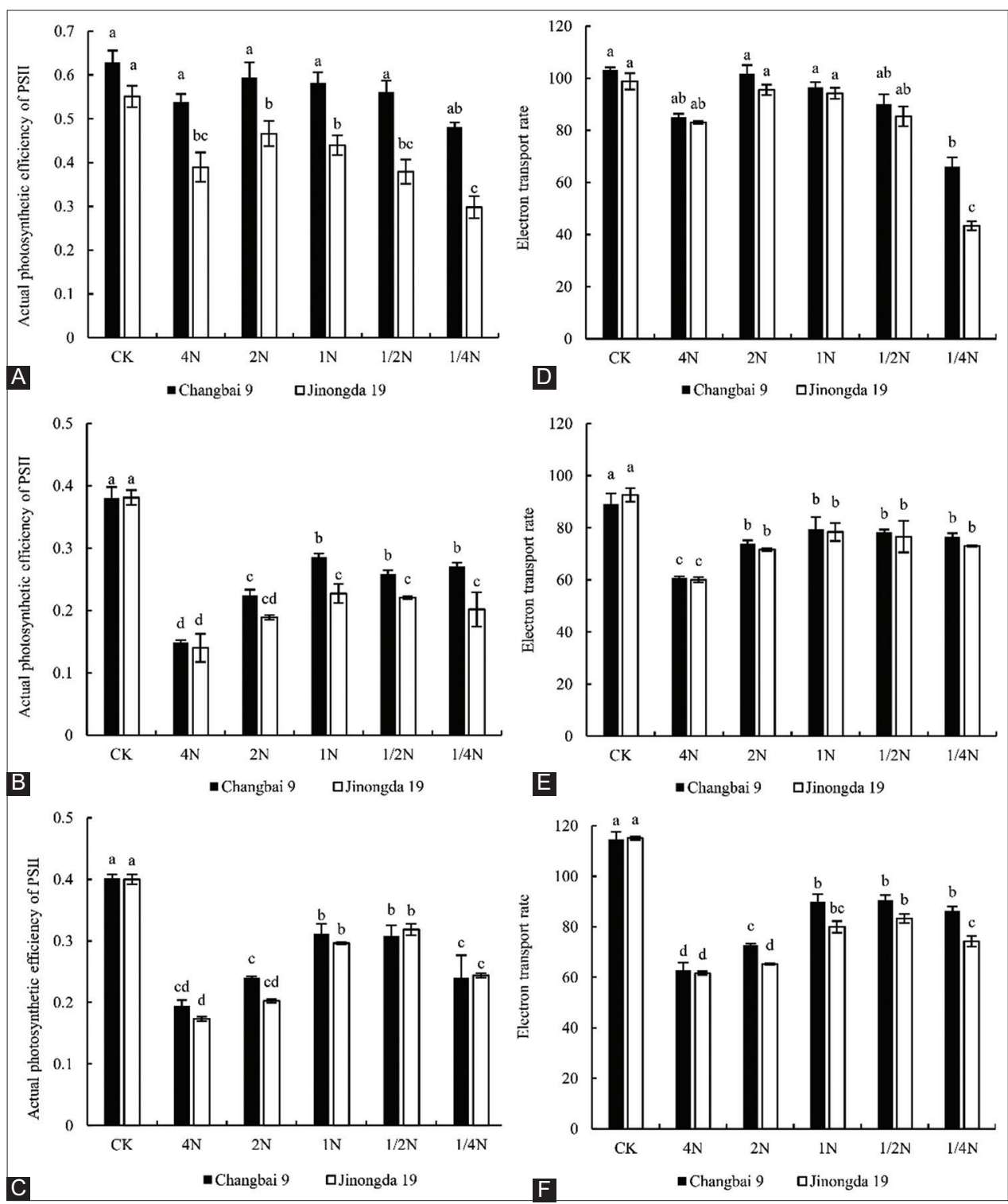

Fig 7. Effect of salt stress on actual photosynthetic efficiency of PSII and electron transport rate of rice leaves for nitrogen levels during different growth periods $(A, C)$ Tillering period; $(B, E)$ Booting period; $(C, F)$ Heading period. Different lowercase letters indicate significant differences $(P<0.05)$.

respectively, under salt stress. $L_{\mathrm{s}}$ had the opposite trend in the above four parameters, except heading period, and the percent increases were the smallest in the $2 \mathrm{~N}, 1 \mathrm{~N}$, and $1 \mathrm{~N}$ treatments during the three respective growth periods.

Therefore, it is speculated that at low nitrogen treatment levels, non-stomatal restriction was the main factor in reducing photosynthesis. With the increase of nitrogen concentration, stomatal restriction was also involved in the decrease of photosynthesis. Compared with the normal nitrogen supply treatment, the high concentrations of nitrogen made the stomata of rice leaves lose their function of opening and closing, which was more harmful to rice leaves. Compared with the control, the variation trend of AMC in rice leaves was similar to that of $P_{\mathrm{N}}, g_{\mathrm{s}}, C_{\mathrm{i}}$, and $E$ during the tillering, booting, and heading periods. RuBP carboxylase is an important regulatory enzyme in carbon metabolism of photosynthesis. It is abundant in rice and mainly exists in the soluble part of chloroplasts. The activity of $\mathrm{R} u \mathrm{BP}$ carboxylase is related to the degree of salt damage to rice. Therefore, it is speculated that with the progress of rice growth and development, reducing the nitrogen supply level can effectively alleviate the harm caused by salt stress to maintain RuBP carboxylase activity in rice so as to decrease salt damage. The results also showed that the $P_{\mathrm{N}}, g_{\mathrm{s}}$ and $E$ of the salt-tolerant rice variety Changbai 9 were higher than that of the salt-sensitive rice variety Jinongda 19 . The change of $C_{i}$ between the two rice varieties did not follow the pattern reflected by the above indexes. This may be due to the decrease of photosynthesis in salt-tolerant rice varieties mainly due to non-stomatal 

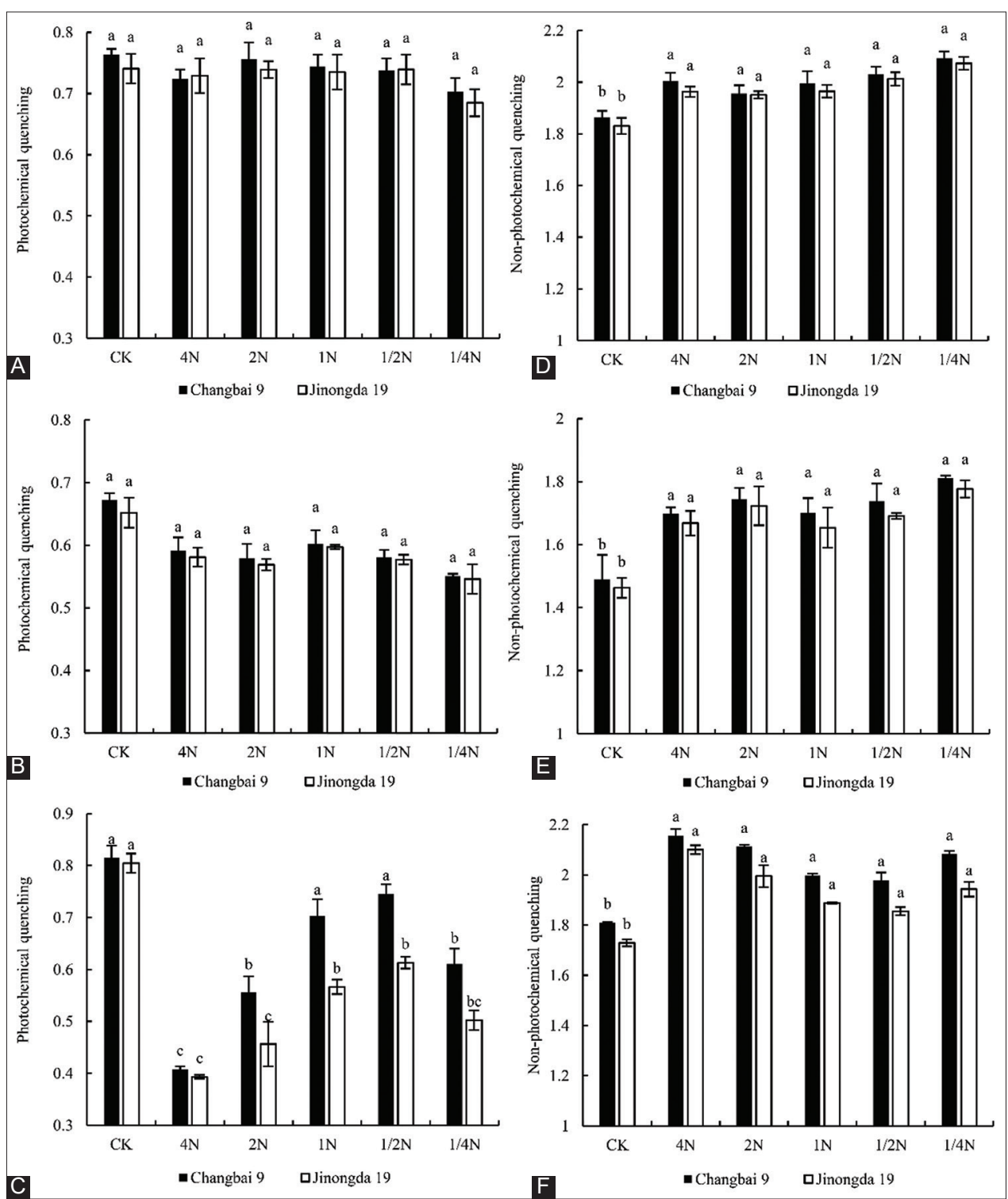

Fig 8. Effect of salt stress on photochemical quenching and non-photochemical quenching of rice leaves for different nitrogen levels during different growth periods $(A, C)$ Tillering period; $(B, E)$ Booting period; $(C, F)$ Heading period. Different lowercase letters indicate significant differences $(P<0.05)$.

restriction. The reason for the decrease of photosynthesis in salt-sensitive rice varieties is that the stomatal limiting factors were added to the non-stomatal limiting factors. It was speculated that RuBP carboxylase activity of salt-tolerant varieties was higher than that of salt-sensitive varieties under salt stress, AMC's trends support this view. This may be due to the physiological advantages of salt-tolerant varieties over salt-sensitive varieties.

\section{Dark adaptation}

If the energy absorbed by the plant photosystem II cannot spread out in time under salt stress, it will render the photosynthetic mechanism useless (Zulfiqar and Shahbaz 2013). The increase of NPQ will have a protective effect on plant leaves against adversity. Some studies found that the appropriate nitrogen concentration could improve the
PSII activity of maize leaves, enlarge the openness of the PSII reaction center and raise the light energy utilization of leaves under high salt stress. Non-photochemical quenching was used to avoid damage to the photosynthetic system II reaction center by means of heat dissipation (Akram et al., 2011). $F_{v} / F_{m}$ was one of the best parameters to determine the inhibited degree of rice photosynthesis. The results of our study indicated that the $\mathrm{F}_{\mathrm{v}} / \mathrm{F}_{\mathrm{m}}$ of two rice varieties showed a downtrend under salt stress. Compared with the control, with an increase of nitrogen concentration in the nutrient solution, the $\mathrm{F}_{\mathrm{v}} / \mathrm{F}_{\mathrm{m}}$ of the two rice varieties leaves decreased significantly during the heading period, while the difference of the salt-sensitive variety from the control was more significant than that of the salt-tolerant variety. During the tillering and booting periods, the $\mathrm{F}_{\mathrm{v}} / \mathrm{F}_{\mathrm{m}}$ of the salt-tolerant variety Changbai 9 
showed no significant difference with the increase in the nitrogen concentration, and showed a significant decrease only in the $1 / 4 \mathrm{~N}$ treatment. The percent decreases of $\mathrm{F}_{\mathrm{v}} / \mathrm{F}_{\mathrm{m}}$ of rice leaves in the $2 \mathrm{~N}, 1 \mathrm{~N}$, and $1 / 2 \mathrm{~N}$ treatments were the smallest during the tillering, booting, and heading periods, respectively, indicating that these nitrogen levels could relieve the injury of the salt stress on the rice photosynthetic system PSII and increased the activity of PSII during the these three periods. This might be due to the accelerated synthesis of chlorophyll in the rice leaves, which improved the efficiency of light energy absorption, conversion, and transmission.

\section{Light adaptation}

The light quantum of the PSII reaction center converted energy mainly through a photochemical pathway (Biao et al., 2013). The results of this study showed that the $\Phi_{\text {PSII }}$ and ETR of the two rice varieties exhibited a lower magnitude of decline decreased significantly under salt stress. Compared with the control, the $\Phi_{\text {PSII }}$ and ETR of the two rice varieties was the smallest in the $2 \mathrm{~N}, 1 \mathrm{~N}$, and $1 / 2 \mathrm{~N}$ treatments during the tillering, booting, and heading periods. This indicated that with the development of rice, damage occurred to the leaf photosynthetic system II under salt stress leading $\Phi_{\text {PSII }}$ and ETR to decrease (Hao et al., 2012). Proper reduction of nitrogen concentration could help protect the photosynthetic system II of rice leaves, improve the PSII activity of rice leaves, and regulate PSII to dissipate the excess light energy to resist the damage to the PSII reaction center.

\section{Quenching}

$\mathrm{q}_{\mathrm{p}}$ reflected the energy used for photochemical electron transfer, while NPQ reflected the energy emitted in the form of heat dissipation that cannot be used for photochemical electron transfer (Akram et al., 2011). The results of this study showed that salt stress caused $\mathrm{q}_{\mathrm{p}}$ to decrease and NPQ to increase in the two rice varieties. With the increase in nitrogen concentration, there was no significant difference between different nitrogen levels in $\mathrm{q}_{\mathrm{p}}$ during the tillering and booting periods. In the heading period, the percent decrease of Changbai 9 in the $1 / 2 \mathrm{~N}$ treatment was the smallest. Therefore, it is speculated that the appropriate reduction of nitrogen supply during the heading period can increase the openness of the PSII reaction center in rice leaves, so as to resist adversity. Compared with the control, NPQ in the $2 \mathrm{~N}, 1 \mathrm{~N}$, and $1 / 2 \mathrm{~N}$ treatments had the smallest percent decrease on tillering, booting and heading periods, indicating that along with the advancement of rice growth period, The demand for nitrogen in rice is decreasing, Increasing the nitrogen supply level under salt stress will aggravate the damage of salt stress to photosystem, During heading stage, nitrogen supply should be reduced appropriately, which is beneficial for rice to resist salt damage.

\section{CONCLUSION}

In conclusion, non-stomatal limitation is the main factor of photosynthesis decreased at low nitrogen level. With the increase of nitrogen concentration, stomatal limitation was also added to the decrease of photosynthesis. Reducing nitrogen supply level can maintain $\mathrm{R} u B P$ carboxylase activity in rice with the advance of rice growth process, thus reducing salt damage. Appropriate nitrogen level can improve the PSII activity of rice leaves under salt stress, increase the degree of PSII reaction center opening, and improve its light energy utilization efficiency. The reduction of nitrogen supply by $50 \%$ at heading stage can alleviate the damage of salt stress on rice PSII and achieve the purpose of resisting adversity. Reducing nitrogen fertilizer by $50 \%$ at heading stage could help rice maintain higher photosynthetic capacity and photosystem II activity under salt stress. It can increase the photosynthetic material production capacity of rice, thus providing effective help for the stable yield of rice under salt environment. This is of great theoretical and practical significance to rice production and breeding of salt-tolerant rice varieties in Jilin Province.

\section{ACKNOWLEDGMENTS}

The authors would like to thank the National key research and development program of China (Project Numbers: 2017YFD0300609, 2018YFD0300207), Technology development program of Jilin Province in China (Project Numbers: 20170203003NY) and Jilin provincial department of science and technology, technology research projects (Project Numbers: 20190301017N Y) for their financial support.

\section{Authors' contribution}

Xu Chen and Li Qian conducted the experiment, analyzed the data and wrote this article. Liu Xiaolong compiled and analyzed the data. Wang Hongjun, Liang Xuanhe provided help for the experimental design and participated in the experiment. Ling Fenglou and Wu Zhihai designed the experiment. Zhang Zhian and Chen Zhanyu provided the financial support for this study and participated in the manuscript processing of this article. All authors read and approved the final manuscript.

\section{REFERENCES}

Akram, M., M. A. Ashraf, M. Jamil, R. M. Iqbal, M. Nafees and M. A. Khan. 2011. Nitrogen application improves gas exchange characteristics and chlorophyll fluorescence in maize hybrids under salinity conditions. Russ. J. Plant Physiol. 58(3): 394-401.

Ali-Dinar, H. M., G. Ebert and P. Lüdders. 1999. Growth, chlorophyll content, photosynthesis and water relations in guava (Psidium guajava L.) under salinity and different nitrogen supply. 
Gartenbauwissenschaft. 64: 54-59.

Ashraf, M. and R. Sultana. 2000. Combination effect of $\mathrm{NaCl}$ salinity and nitrogen form on mineral composition of sunflower plants. Biol. Plant. 43(4): 615-619.

Biao, G., D. Wen, K. V. Langenberg, M. Wei, F. J. Yang, Q. H. Shi and X. F. Wang. 2013. Comparative effects of $\mathrm{NaCl}$ and $\mathrm{NaHCO}_{3}$ stress on photosynthetic parameters, nutrient metabolism, and the antioxidant system in tomato leaves. Sci. Hortic. 157(20): 1-12.

Brandner, S. J. C. and M. E. Salvucci. 2002. Sensitivity of photosynthesis in a C4 plant, maize, to heat stress. Plant Physiol. 129(4): 1773-1780.

Chakrabarti, N. and S. Mukherji. 2003. Effect of phytohormone pretreatment on nitrogen metabolism in Vigna radiata under salt stress. Biol. Plant. 46(1): 63-66.

Chaum, S. and C. Kirdmanee. 2009. Effect of salt stress on accumulation, photosynthetic ability and growth characters in two maize cultivars. Pak. J. Bot. 41(1): 87-98.

Chaves, M. M., J. Flexas and C. Pinheiro. 2009. Photosynthesis under drought and salt stress: Regulation mechanisms from whole plant to cell. Ann. Bot. 103(4): 551-560.

Chi, C. M. and Z. C. Wang. 2010. Characterizing salt-affected soils of songnen plain using saturated paste and 1: 5 soil-to-water extraction methods. Arid Land Res. Manage. 24: 1-11.

da Silva, E. C., R. J. M. Nogueira, F. P. de Araújo, N. F. de Melo and N. A. D. de Azevedo. 2008. Physiological responses to salt stress in young umbu plants. Environ. Exp. Bot. 63: 147-157.

Gamett, T., V. Conn and B. Kaiser. 2009. Root based approaches to improving nitrogen use efficiency in plants. Plant Cell Environ. 32(9): 1272-1283.

Golldack, D., C. Li, H. Mohan and N. Probst. 2014. Tolerance to drought and salt stress in plants: Unraveling the signaling networks. Front. Plant Sci. 5(151): 1-10.

Hao, H., H. Zhang, J. M. Chu and X. M. Zhang. 2012. Leaf chlorophyll fluorescence effect of different rice (Oryza sativa L.) genotypes under salt stress. J. Comput. Theor. Nanosci. 11(1): 706-709.

Hoai, N. T. T., I. S. Shim, K. Kobayashi and U. Kenji. 2003. Accumulation of some nitrogen compounds in response to salt stress and their relationships with salt tolerance in rice (Oryza sativa L.) seedlings. Plant Growth Regul. 41(2): 159-164.

Liu, X. L., C. Xu, K. Z. Xu, J. J. Cui, Z. A. Zhang, F. L. Ling, J. H. An and Z. H. Wu. 2015. Effects of salt stress on photosynthetic characteristics and some physiological traits of rice varieties at different nitrogen levels. J. S. China Agric. Univ. 36(2): 6-12.

Lu, C. M., N. W. Qu, Q. T. Lu, B. S. Wang and T. Y. Kuang. 2002. Does salt stress lead to increased susceptibility of photosystem II to photoinhibition and changes in photosynthetic pigment composition in halophyte Suaeda salsa grown outdoors? Plant Sci. 163: 1063-1068.

Munns, R. and M. Tester. 2008. Mechanisms of salinity tolerance. Annu. Rev. Plant Biol. 59: 651-681.

Netondo, G. W., J. C. Onyango and E. Beck. 2004. Sorghum and salinity: II. Gas exchange and chlorophyll fluorescence of sorghum under salt stress. Crop Sci. 44(3): 806-811.

Parida, A. K. and A. B. Das. 2005. Salt tolerance and salinity effects on plants: A review. Ecotoxicol. Environ. Saf. 60: 324-349.

Serrano, R., J. M. Mulet, G. Rios, J. A. Marquez, I. F. de Larrinoa, M. P. Leube, I. Mendizabal, A. Pascual-Ahuir, M. Proft, R. Ros and C. Montesinos. 1999. A glimpse of the mechanisms of ion homeostasis during salt stress. J. Exp. Bot. 50: 10231036.

Siddiqui, M.H., F. Mohammad, M. N. Khan, M. H. Al-whaibi and A. H. A. Bahkali. 2010. Nitrogen in relation to photosynthetic and accumulation of osmoprotectant and nutrients in Brassica genotyped grown under salt stress. J. Integr. Agric. 9(5): 671-680.

Soussi, M., A. Ocaña and C. Lluch. 1998. Effects of salt stress on growth, photosynthesis and nitrogen fixation in chick-pea (Cicer arietinum L.). J. Exp. Bot. 49(325): 1329-1337.

Summart, J., P. Thanonkeo, S. Panichajakul, P. Prathepha and M. T. McManus. 2010. Effect of salt stress on growth, inorganic ion and proline accumulation in Thai aromatic rice, Khao Dawk Mali 105, callus culture. Afr. J. Biotechnol. 9: 145-152.

Zulfiqar, S. and M. Shahbaz. 2013. Modulation of gas exchange parameters and photosystem II activity of canola (Brassica napus L.) by foliar-applied triacontanol under salt stress. Agrochimica. 57(3): 193-200. 